\title{
Study of cubic GaN clusters in hexagonal GaN layers and their dependence with the growth temperature
}

\author{
J. Laifi ${ }^{\text {a, }}{ }^{*}$, N. Chaaben ${ }^{\text {a }}$, Y. El Gmili ${ }^{\text {b }}$, J.P. Salvestrini ${ }^{\text {c }}$, A. Bchetnia ${ }^{\text {a }}$, B. El Jani ${ }^{\text {a }}$ \\ a Unité de Recherche sur les Hétéro-Epitaxies et Applications, Faculté des Sciences de Monastir 5019, Université de Monastir, Tunisia \\ b CNRS, UMI 2958 Georgia Tech-CNRS, 57070 Metz, France \\ ${ }^{\mathrm{c}}$ Université de Lorraine, Centrale Supelec, LMOPS, EA4423, 57070 Metz, France
}

The inclusion of cubic phase in MOVPE-grown hexagonal GaN on GaAs substrate and its dependence with the growth temperature are investigated by high-resolution X-ray diffraction (HR-XRD), scanning electron microscopy (SEM), atomic force microscopy (AFM) and cathodoluminescence (CL). It is observed that the GaN layers surface exhibits 3D-grains structure. The density and shape of these grains are largely dependent on the growth temperature. HR-XRD study reveals the presence of cubic GaN clusters in the hexagonal GaN layer. Using CL we show that the cubic inclusions are not localized at the substrate/epilayer interface but propagate throughout the film.

\section{Introduction}

GaN wide band gap semiconductor and its alloys are useful materials for the fabrication of devices operating in blue and ultraviolet spectral regions [1,2]. The most stable crystalline structure of GaN is the hexagonal structure (h-GaN), thus devices including light emitting diodes are based on h-GaN. High-quality h-GaN films and heterostructures are usually grown either by metal organic chemical vapor deposition (MOVPE) or by molecular beam epitaxy (MBE) on sapphire $\left(\mathrm{Al}_{2} \mathrm{O}_{3}\right)$ and $6 \mathrm{H}-\mathrm{SiC}$ substrates [3,4]. However, hGaN layer shows spontaneous and piezoelectric-induced polarization [5], which is generally a drawback for devices [6]. c-GaN results in more isotropic physical properties without detrimental polarization effects. Thus, growing GaN in pure cubic structure (c-GaN) or mixed cubic-hexagonal structure, as reported by Saengkaew et al. [7], is promising to achieve non-polar or semi-polar GaN layers. In addition, c-GaN layers offer easy cleavage along (001) facet orientation, superior electronic proprieties, and high doping efficiencies [8-10] as compared to h-GaN layers.

It has been reported that c-GaN layers can be grown on different substrates such as (001) GaAs [11], (001) Si [12] and 3C-SiC [13], but

\footnotetext{
* Corresponding author

E-mail address: amor.bchetnia@fsm.rnu.tn (J. Laifi).
}

the most commonly employed substrate for this is GaAs (001). GaAs substrates are preferred because of their advantage for device fabrication due to easy cleaving, etching, and ohmic contact realization. Nevertheless, issues such as large lattice mismatch between the GaAs substrate and GaN layer, small difference between the formation energy of the two phases (hexagonal and cubic), and high decomposition rate of GaAs substrate in ammonia $\left(\mathrm{NH}_{3}\right)$ at temperature above $700{ }^{\circ} \mathrm{C}$ lead to high structural quality c-GaN layers growth challenging [14-17]. To improve the growth of c-GaN on GaAs substrate it is necessary to go further in the understanding of the link between the formation of the cubic phase and the deposition conditions. In a previous study, we have shown that a significant improvement of the GaN layer structural quality can be achieved using substrate nitridation and low temperature buffer layer deposition before the growth of the epitaxial GaN layer at higher temperature. These steps prevent the decomposition of the GaAs substrate surface and enhance the GaN nucleation to initiate the growth of the GaN layer [18]. We also have shown, with other authors [19-21], that to obtain and stabilize the cubic phase, the deposition conditions (V/III ratio, growth temperature, GaAs substrate orientation) have to be optimized. In this paper, we focus on the growth and characterization of GaN layers on GaAs (001) substrate and we study, using in-situ reflectivity measurements, scanning electron microscopy (SEM), atomic force microscopy (AFM), high-resolution X-ray diffraction (HR-XRD) and 
cathodoluminescence $(\mathrm{CL})$, the formation of cubic inclusions in the hexagonal GaN layer as function of the growth temperature in the temperature range of $700-850{ }^{\circ} \mathrm{C}$.

\section{Experimental details}

GaN layers were grown by atmospheric pressure metalorganic vapor phase epitaxy (AP-MOVPE) on (001) GaAs substrate. Ammonia $\left(\mathrm{NH}_{3}\right)$ and Trimethylgallium (TMG) were used as a precursor of nitrogen (N) and gallium (Ga), respectively. $\mathrm{H}_{2}$ was used as a carrier gas with a total flow rate of $2 \mathrm{slm}$ (standard liter per minute). The growth process starts by nitridation of GaAs substrate under $\mathrm{NH}_{3}$. This step is followed by the growth of a $\mathrm{GaN}$ buffer layer at the same growth temperature used during the nitridation process. Four different GaN layers were then grown at different growth temperatures on top of the buffer layer. The different samples are labeled ${ }^{\#} S_{1},{ }^{\#} S_{2},{ }^{\#} S_{3}$ and ${ }^{\#} S_{4}$ and correspond to growth temperature of $700,750,800$ and $850{ }^{\circ} \mathrm{C}$, respectively. Details of the growth process of the samples can be found elsewhere [21]. The growth was in-situ monitored by single wavelength $(\lambda=632.8 \mathrm{~nm}) \mathrm{He}-\mathrm{Ne}$ laser reflectometry operated at normal incidence. The surface morphology is characterized by scanning electron microscopy (SEM) and atomic force microscopy (AFM) at a scale of $5 \times 5 \mu \mathrm{m}^{2}$. Xray diffraction measurements were performed using a highresolution diffractometer equipped with a fourfold Ge ( $\left.\begin{array}{lll}2 & 2 & 0\end{array}\right)$ monochromator, delivering a pure $\mathrm{CuK} \alpha_{1}$ line of wavelength $(\lambda=0.154 \mathrm{~nm})$. Room temperature depth-resolved $\mathrm{CL}$ investigations are performed in a digital scanning electron microscope. Emitted light is detected via a parabolic mirror collector and analyzed by a spectrometer with a focal length of $320 \mathrm{~mm}$ using a 1200 grooves $\mathrm{mm}^{-1}$ grating and a spectral resolution of $0.06 \mathrm{~nm}$. The signal is then recorded by a liquid $\mathrm{N}_{2}$-cooled Horiba Jobin Yvon Instruments Symphony $1024 \times 256$ CCD detector.

The depth profile of residual impurities along the growth direction was obtained by secondary ion mass spectroscopy SIMS. The calibration of elemental concentration for this instrument was done by using relative sensitivity factors (RSFs) derived from the analysis of a GaN standard with known doses of impurity implants. SIMS measurements of negative and positive secondary ions were performed with the ultra-low energy conditions of $3 \mathrm{keV}$ primary ion bombardment impact energy by Cesium and Oxygen respectively.

\section{Results and discussion}

Fig. 1 shows the reflectance curves recorded as function of the time during the growth of the GaN layers on $\mathrm{GaAs}(001)$ substrate for temperature varying between $700{ }^{\circ} \mathrm{C}$ and $850{ }^{\circ} \mathrm{C}$. Four steps can be distinguished: (i) nitridation of GaAs substrate at $550{ }^{\circ} \mathrm{C}$, (ii) low temperature $\left(550{ }^{\circ} \mathrm{C}\right)$ growth of $65 \mathrm{~nm}$ thick GaN buffer layer, (iii) temperature ramp up, (iv) GaN layer deposition in the temperature range of $700-850{ }^{\circ} \mathrm{C}$. For all samples, as expected, the reflectance signal corresponding to steps (i-iii) does not exhibit any difference but differs in step (iv). In this step, sample ${ }^{\#} \mathrm{~S}_{2}$, for which the GaN layer is grown at $750^{\circ} \mathrm{C}$, is characterized by regular oscillations of the reflectance signal with a nearly constant (or slightly decreasing) amplitude. In contrary, for the other samples the amplitude of the oscillations in the reflectance signal clearly decreases with time. The largest oscillation damping is observed in sample ${ }^{\#} \mathrm{~S}_{4}$ for which the $\mathrm{GaN}$ layer is grown at the highest temperature $\left(850{ }^{\circ} \mathrm{C}\right)$. The damping of the oscillation in the reflectance signal is attributed to changes in the surface morphology of GaN films and can be estimated by calculating the damping rate $r_{d}$ using the following equation [18]:

$\left.r_{d}=\log \frac{R_{\max (i-1)}}{R_{\max (i)}}\right)$

where (i) is the order of the reflectance maximum $\left(R_{\max }\right)$. The inset

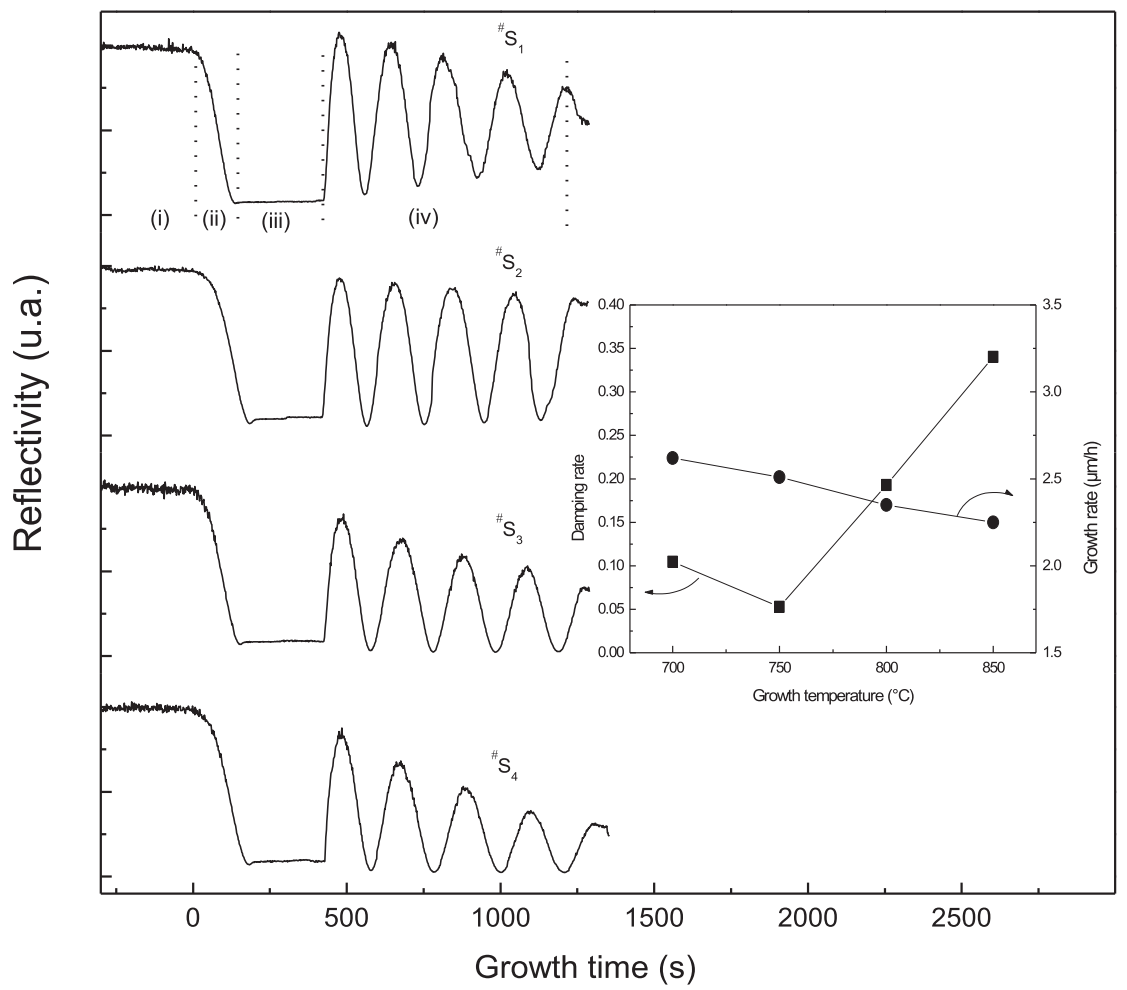

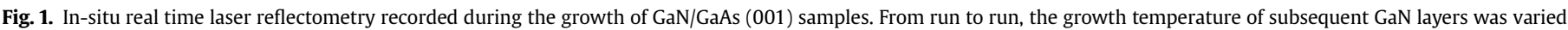
from $700{ }^{\circ} \mathrm{C}\left({ }^{\#} \mathrm{~S}_{1}\right)$ to $850{ }^{\circ} \mathrm{C}\left({ }^{\#} \mathrm{~S}_{4}\right)$. Inset shows the plot of damping rate and growth rate versus growth temperature. 

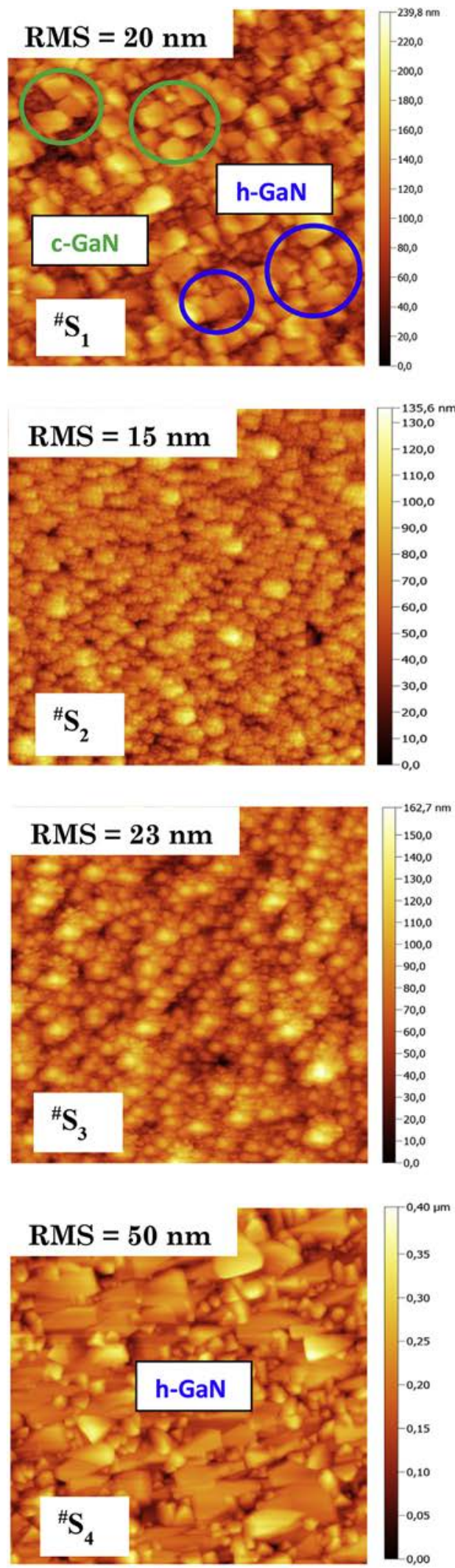

(a)
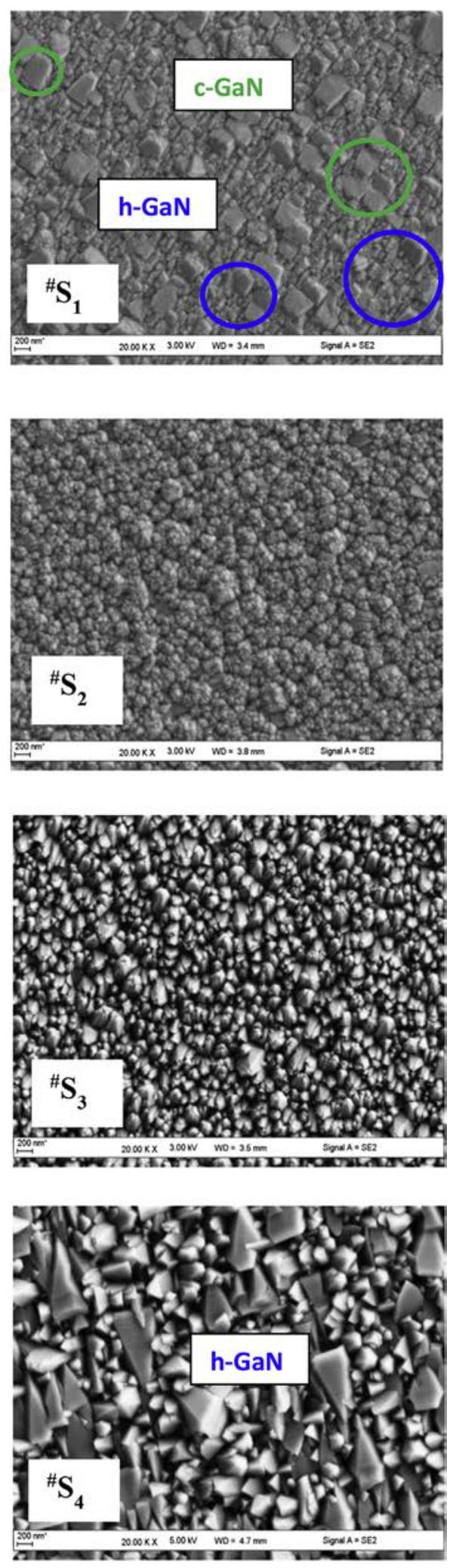

(b)

Fig. 2. (a): AFM images of the four subsequent GaN layers ${ }^{\#} S_{1},{ }^{\#} S_{2},{ }^{\#} S_{3}$ and ${ }^{\#} S_{4}$. (b): SEM images indicating the crystalline properties of the four samples. 
of Fig. 1 shows the dependence with growth temperature of both the damping rate and growth rate (derived from the number of oscillations in the reflectance signal). Since a weak value of $r_{d}$ corresponds to a low surface roughness, it is clear that the sample ${ }^{\#} \mathrm{~S}_{2}$ grown at $750{ }^{\circ} \mathrm{C}$ exhibits the lowest surface roughness. In contrary, for the sample ${ }^{\#} S_{4}$ grown at $850{ }^{\circ} \mathrm{C}, \mathrm{r}_{\mathrm{d}}$ is seven times higher indicating a large surface roughening of the GaN layer. As shown also in the inset of Fig. 1, in opposite to the large dependence of the surface roughness with the growth temperature, the growth rate decreases slowly with the increase of the growth temperature. To go further, the morphological properties of the four GaN samples recorded using both AFM and SEM are shown in Fig. 2(a) and (b), respectively. It is clear that all the samples exhibit poly-crystalline structure features with three dimensional grains structure. At a growth temperature of $700{ }^{\circ} \mathrm{C}\left({ }^{\#} \mathrm{~S}_{1}\right)$, we can notice the presence on
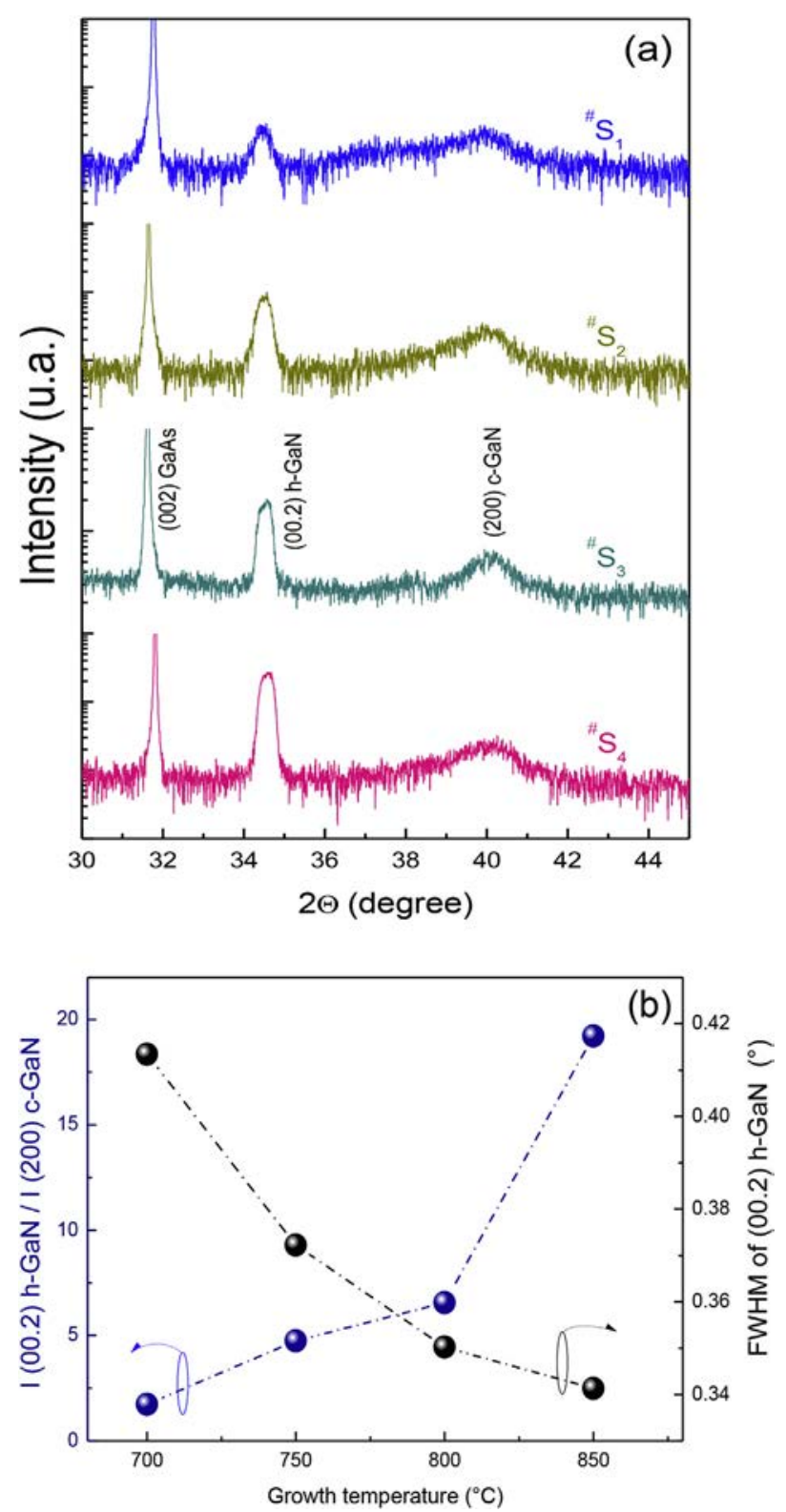

Fig. 3. (a): XRD patterns of GaN samples with subsequent layers grown at $700{ }^{\circ} \mathrm{C}\left({ }^{\#} \mathrm{~S}_{1}\right)$, $750{ }^{\circ} \mathrm{C}\left({ }^{\#} \mathrm{~S}_{2}\right), 800{ }^{\circ} \mathrm{C}\left({ }^{\#} \mathrm{~S}_{3}\right)$ and $850{ }^{\circ} \mathrm{C}\left({ }^{\#} \mathrm{~S}_{4}\right)$. (b): variation of the ratio of $(00.2) \mathrm{h}-\mathrm{GaN} \mathrm{X}-$ ray peak intensity to the (200) c-GaN peak intensity and the FWHM of the (00.2) hGaN peak as a function of growth temperature. the surface of few grains exhibiting in some extent a cubic symmetry as well as a high density of pyramid-like structures which reveal the presence of an hexagonal phase. This surface morphology, with a RMS of $20 \mathrm{~nm}$, could correspond to that of a hexagonal GaN layer containing cubic GaN domains. For higher growth temperature $\left(750-800^{\circ} \mathrm{C}\right)$, the surface morphology of ${ }^{\#} \mathrm{~S}_{2}$ and ${ }^{\#} S_{3}$ shows the formation of highly dense hexagonal GaN grains with an average size of about $300 \mathrm{~nm}$ and $500 \mathrm{~nm}$, as estimated from AFM data for ${ }^{\#} S_{2}$ and ${ }^{\#} S_{3}$, respectively. At the highest growth temperature $\left(850{ }^{\circ} \mathrm{C},{ }^{\#} \mathrm{~S}_{4}\right)$, the surface of the GaN layer becomes very rough $(\mathrm{RMS}=50 \mathrm{~nm})$ with an island like structure formed by randomly distributed and oriented crystallites having non regular shapes and different sizes (most of them larger than $1 \mu \mathrm{m}$ ).

To go further and assess the crystalline quality of the samples, Fig. 3(a) shows the XRD spectra recorded for the different samples. In addition to the peak corresponding to the (002) plane of the GaAs substrate, two other diffraction peaks can be seen at $2 \theta=34.46^{\circ}$ and $39.82^{\circ}$ which can be attributed to the (00.2) and (200) planes of the hexagonal and cubic phases, respectively. Data of Fig. 3(a) allows plotting the intensity ratio of the (00.2) h-GaN peak and (200) c-GaN peak as well as the FWHM of the (00.2) hGaN peak versus the growth temperature of the GaN layer. It can be seen in Fig. 3(b) that the intensity ratio increases with the growth temperature increase. This means that the fraction of hexagonal phase (cubic phase) in the GaN layer increases (decreases) with the increase of the growth temperature as evidenced by Tachibana et al. [16]. As shown in Fig. 3(b), the decrease of the FWHM of the (00.2) h-GaN peak with the increase of the growth temperature indicates also that the alignment of the crystallites with respect to $<00.1>$ axis of the wurtzite structure increases for high growth temperature. This is consistent with SEM and AFM observations, which show packed GaN crystallites having basal and prismatic facets for a growth temperature of $850^{\circ} \mathrm{C}$ and cubic grains for lower growth temperature. This is also in agreement with the works of Suandon et al. [20] and Strite et al. [22] who have shown that the mechanism of transformation of GaN from cubic to hexagonal phase and its dependence with the growth temperature is related

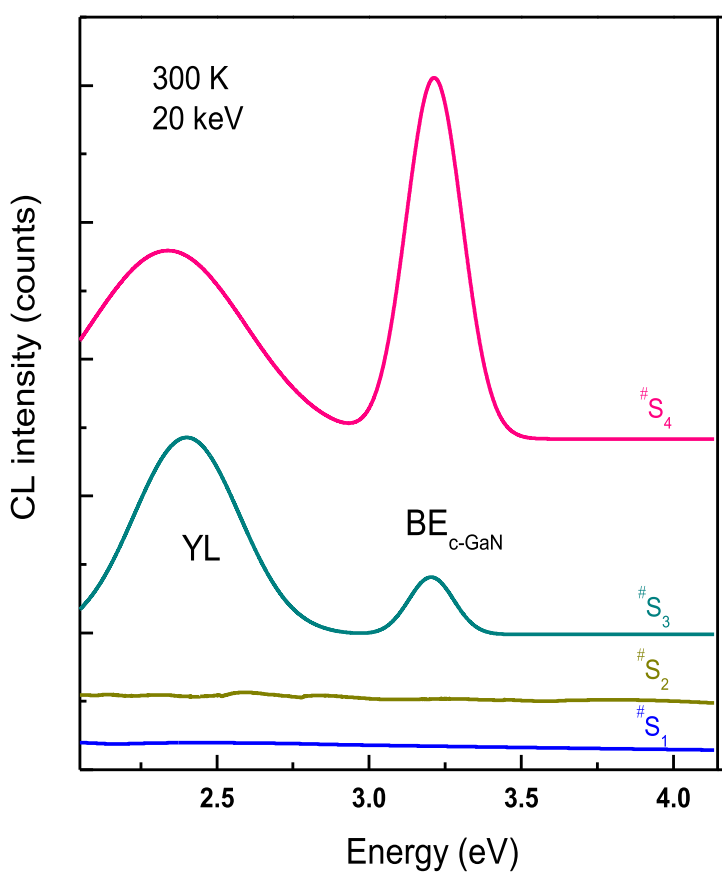

Fig. 4. Room temperature CL spectra for the four samples with ${ }^{\#} S_{1},{ }^{\#} S_{2},{ }^{\#} S_{3}$ and ${ }^{\#} S_{4}$. 
to hexagonal stacking faults generation at the GaN/GaAs interface. Once stacking faults have been generated in some localized areas, the GaN cubic phase turns into hexagonal one, leading to the formation of hexagonal grains at the top surface of the layer.

Fig. 4 shows the CL spectra of the different GaN samples. The measurement were done at room temperature using a focused electron beam with a beam energy of $20 \mathrm{keV}$, allowing the generation of excited photons from a zone which is $0.55 \mu \mathrm{m}$ in depth in the GaN epilayer. As it can be seen, no CL signal is observed for samples ${ }^{\#} S_{1}$ and ${ }^{\#} S_{2}$ probably due to the very low structural quality of these samples as revealed by the XRD spectra shown in Fig. 3(a). ${ }^{\#} \mathrm{~S}_{3}$ and ${ }^{\#} \mathrm{~S}_{4}$ exhibit a sharp near-band-edge luminescence peak at $3.21 \mathrm{eV}$ which is attributed to cubic GaN [23] and whose intensity is larger in ${ }^{\#} S_{4}$ than in ${ }^{\#} S_{3}$ as well as a broad luminescence band attributed to the well-known deep level emission at around $2.40 \mathrm{eV}$ (YL band). The origin of this band most often associated with the transition from a shallow donor to deep acceptor produced by the residual impurities involved in the GaN samples during growth [24-26]. In order to identify the presence of possible contaminants impurities, SIMS analysis was performed. Fig. 5 shows the residual incorporated impurities depth profile in GaN layer grown at $850{ }^{\circ} \mathrm{C}$. Carbon (C), Oxygen (O), Silicon (Si), Hydrogen $(\mathrm{H})$ and Magnesium $(\mathrm{Mg})$ elements were detected with different amounts. The SIMS data near the top surface (tens of $\mathrm{nm}$ ) are in the limits of equilibration distance and should be disregarded. After this interfacial region, we note a flat impurities profile, which indicate a stable control of the growth parameters during the epitaxy process. The sources of these impurities are numerous include the carrier gases, air exposure during wafer loading and the methyl groups of the TMG. The concentrations of different impurities examined in this sample are at the same level with those reported in the literature for good layers qualities [27-30]. At the GaN/GaAs interface, we note an increase of the residual impurities concentration. This behavior could be explained by the fact that the disordered interface region acts as a gettering site for dopant and impurities, especially with strain due thermal expansion mismatch. The intensity maximum ratio $I_{\mathrm{YL}} / I_{\mathrm{BE}}$ of the deep band luminescence (YL) and near band-edge (BE) luminescence peak of ${ }^{\#} S_{3}$ is seven times greater than that of ${ }^{\#} S_{4}$ indicates a much better crystalline quality of the GaN layer in ${ }^{\#} S_{4}$ in agreement with XRD measurements shown in Fig. 2. Although XRD measurements show clear evidence of the GaN hexagonal phase in samples grown at $800{ }^{\circ} \mathrm{C}$ and $850{ }^{\circ} \mathrm{C}$, unexpectedly, none of the $\mathrm{CL}$ spectra shows significant luminescence band corresponding to the hexagonal phase of GaN (3.43eV). In view of our measurements, we

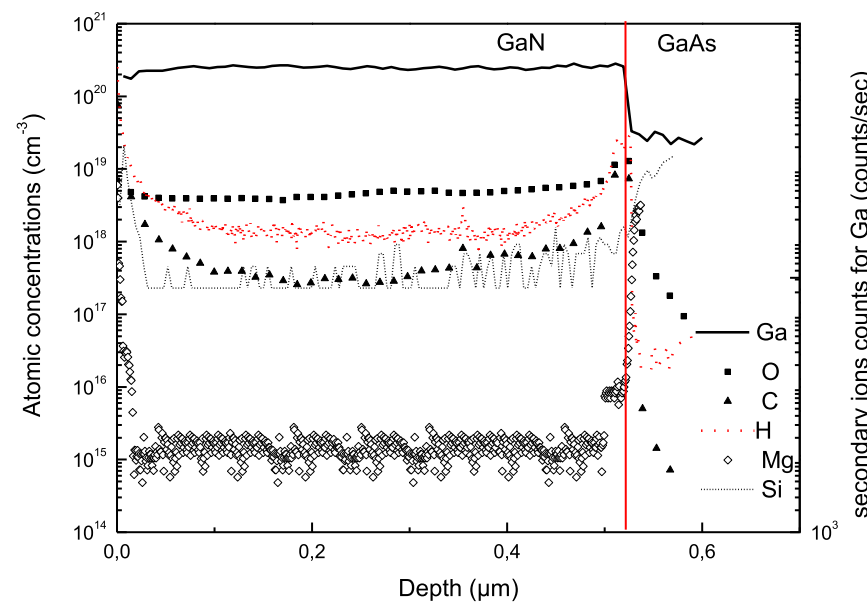

Fig. 5. SIMS profiles of residual impurities in GaN grown layer at $850^{\circ} \mathrm{C}$. propose the following alternative interpretation. The cubic phase likely to be formed by stacking faults at the layer substrate interface is responsible for the emission localized at $3.21 \mathrm{eV}$ in the CL spectrum. It is suggested that since c-GaN has lower band gap than h$\mathrm{GaN}(\Delta \mathrm{Eg}=0.2 \mathrm{eV})$ the inclusions can trap photo-generated carriers $[31,32]$. Then, they will serve as very efficient recombination sites in the expense of the dominant h-GaN phase. Thus, although film is predominantly h-GaN, as shown by SEM and HR-XRD, the emission from c-GaN inclusions dominates the CL spectrum.

Fig 6 shows large area room-temperature depth resolved CL spectra recorded in sample ${ }^{\#} \mathrm{~S}_{4}$ for an electron beam energy varying from 5 to $25 \mathrm{keV}$. With increasing electron beam energy, the CL intensity (BE and YL) increase from 5 until $20 \mathrm{keV}$, above decreases towards the GaN/GaAs interface. The point of maximum excitation (20 keV) produces a maximum of luminescence intensity. The question remains open whether such enhancement of the CL intensity results from accumulation of donor species at interfaces or (for YL) indicates also an increase of impurities concentration in the interface region. For an electron beam energy ranging from 20 to $25 \mathrm{keV}$, the region of maximum energy loss moves from the near surface to the near interface region, as shown in the upper scale of Fig. 7(a). Therefore, the decrease of the CL intensity towards the interface is correlated with the deterioration of the crystalline quality of material. This behavior has been observed by many authors. Godlewski et al. [33] have shown a strong decrease of the CL intensity of GaN close to the interface regions by depth profiling CL studies in MOCVD grown InGaN/GaN/sapphire samples. Martinez et al. [34] have observed a depth variation of intensity of BE emission, donor-acceptor pair (DAP), and YL transitions with beam
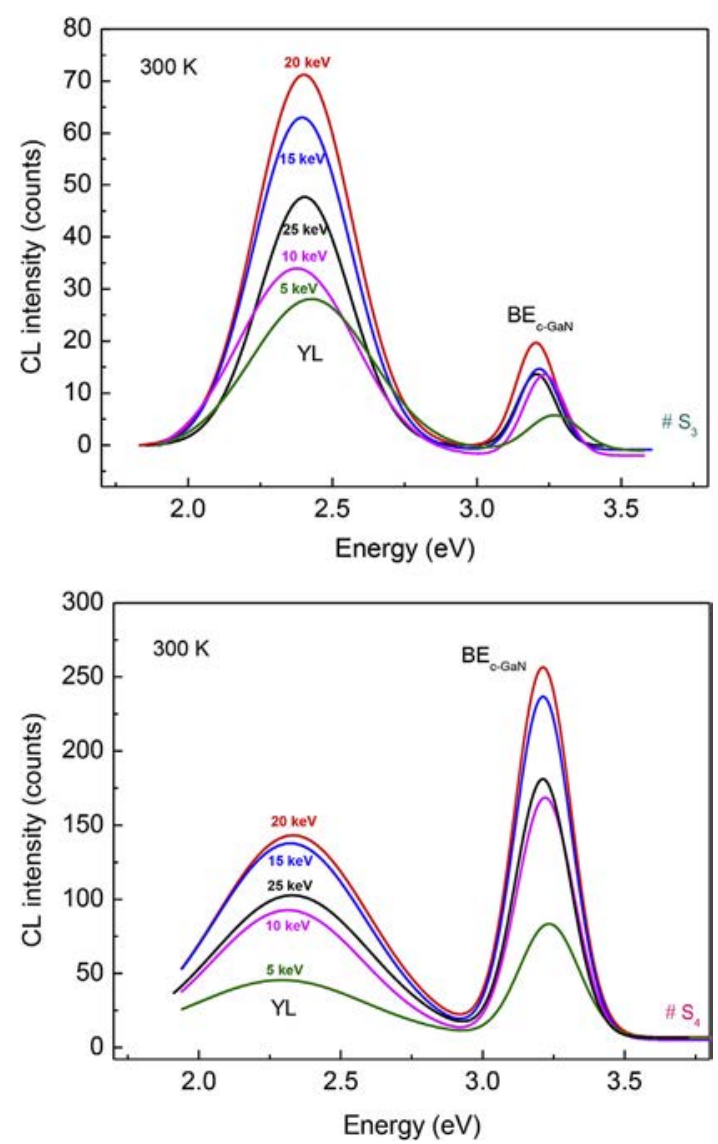

Fig. 6. Room temperature CL spectra acquired from the GaN samples with ${ }^{\#} \mathrm{~S}_{3}$ and ${ }^{\#} \mathrm{~S}_{4}$ at different electron beam energies. 

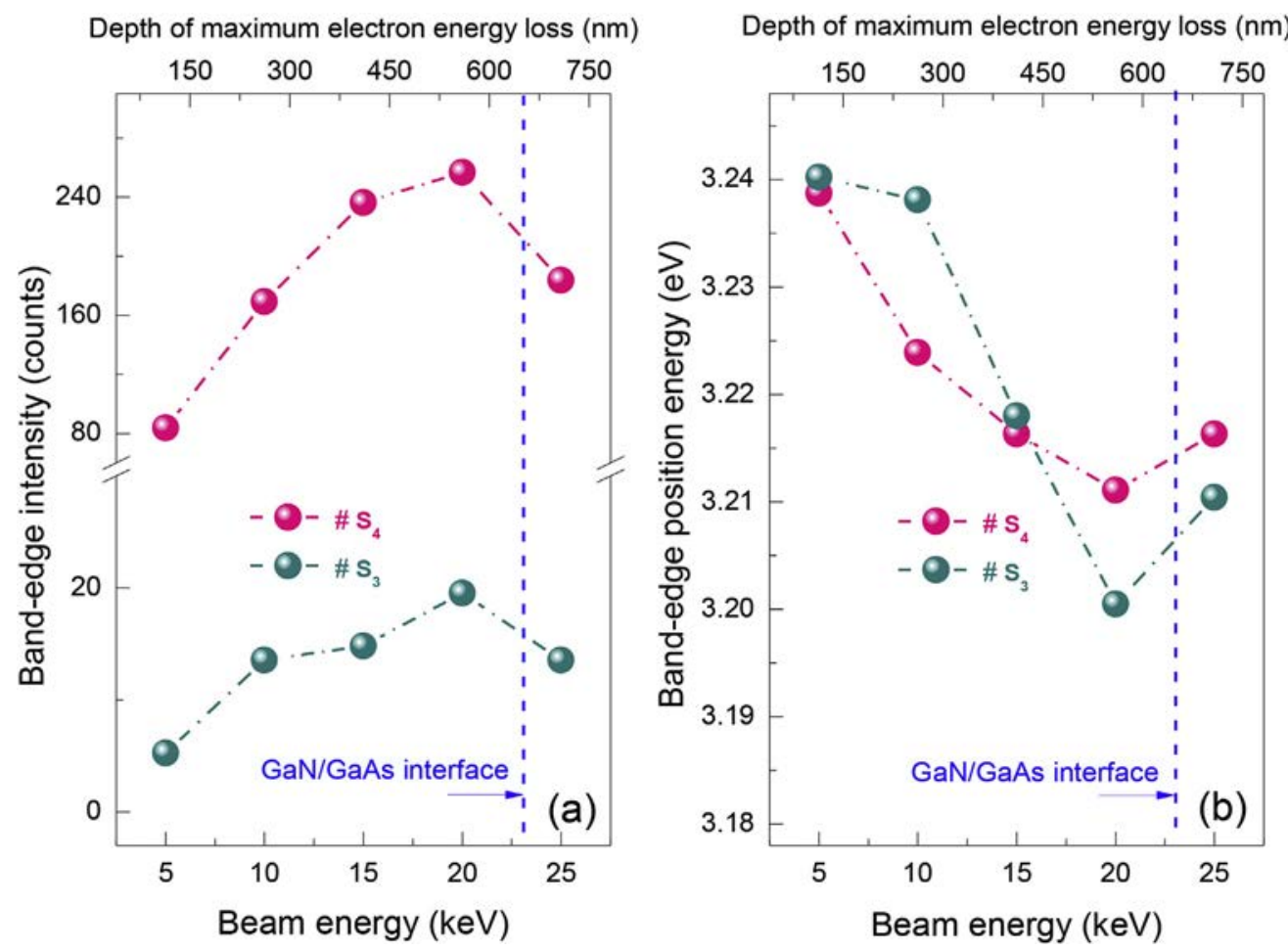

Fig. 7. (a): ${ }^{\#} S_{3}$ and ${ }^{\#} S_{4}$ BE peaks intensities and (b) BE peaks position energy variations vs. the electron beam energy and the depth of maximum energy loss.

energies. Moreover, we observe red-shift by $40 \mathrm{meV}$ and $27 \mathrm{meV}$ of CL BE emissions of ${ }^{\#} S_{3}$ and ${ }^{\#} S_{4}$, respectively, with increasing beam energy from $5 \mathrm{keV}$ to $20 \mathrm{keV}$ (Fig. 7(b)). We attribute the decrease of energy position (red-shift) to the shrinkage of band energy caused by the electron beam heating. The red-shift behavior is in accordance with the results observed by knobloch et al. [35] for the band-edge luminescence of GaN, which they explained by internal absorption effects. When the electron beam energy increases from 20 to $25 \mathrm{keV}$, the BE peaks show a slight shift to higher photon energy by $10 \mathrm{meV}$ and $5 \mathrm{meV}$ for ${ }^{\#} \mathrm{~S}_{3}$ and ${ }^{\#} \mathrm{~S}_{4}$, respectively. It is clear that at this beam energy range, the CL comes mostly from the GaN/ GaAs interface areas. The lattice mismatch between GaN and GaAs $\sim 20 \%$ is accommodated by the internal strains and the formation of large number of stacking faults [36]. This can play a role on the depth variation and the slight blue-shift of the $\mathrm{BE}$ emission observed in our samples. For GaN/Si structures, Ben Nasr et al. [37] have reported a blue shifts between 9 and $15 \mathrm{meV}$ of the BE peak for a beam energy range of 5-20 keV. They attributed these shifts to the increase of the energy gap induced by the strain effects. Other groups $[38,39]$ relate this behavior to confinement and or piezoelectric field effects.

We can draw very important information from the study of depth resolved $\mathrm{CL}$ spectra about the localization of c-GaN inclusions. Therefore, the presence of the c-GaN BE-emission in all CL spectra obtained for different beam energies proves that the c-GaN inclusions, whose presence is demonstrated by X-ray diffraction are not localized at the substrate/epilayer interface but propagate throughout the film.

\section{Conclusion}

The presence of GaN crystallites with some degree of cubic and hexagonal symmetries, as demonstrated by AFM and MEB measurements, suggests the existence of cubic and hexagonal phases in GaN layers grown on (001) GaAs substrates by AP-MOVPE. This result agrees with the XRD analysis. In growth temperature range of $700-850^{\circ} \mathrm{C}$, a transformation takes places from (200) cubic to (00.2) hexagonal GaN phases. The increase of $\mathrm{I}_{(00.2)}$ h-GaN $/ \mathrm{I}_{(200)} \mathrm{c}-$ GaN ratio as a function of growth temperature means that the hexagonal phase fraction in GaN layer as well as the alignment of the crystallites with respect to $<00.1>$ axis of the wurtzite structure increase for high growth temperature. Although XRD measurements show clear evidence of the GaN hexagonal phase in samples grown at $800^{\circ} \mathrm{C}$ and $850^{\circ} \mathrm{C}$, unexpectedly, none of the $\mathrm{CL}$ spectra shows significant luminescence band corresponding to the hexagonal phase of GaN located at $3.43 \mathrm{eV}$. This result can be explained by the spatial separation of photo-generated carriers and their localization at lower band gap energy of cubic GaN. These cubic clusters, which has lower band gap energy than h$\mathrm{GaN}(\Delta \mathrm{Eg}=0.2 \mathrm{eV})$, will serve as very efficient recombination sites in the expense of the dominant h-GaN phase. These results enhance the expectation of applying zinc blend group-III to lightemitting devices.

\section{References}

[1] B. Gill III, Nitride Semiconductors and Their Modern Devices, Oxford University Press, UK, 2013.

[2] S. Nakamura, G. Fasol, The Blue Laser Diode, Springer, 1997.

[3] H.X. Jiang, J.Y. Lin, Opto-electronics Review, vol. 10, 2002, p. 271.

[4] O. Brandt, R. Muralihadharan, A. Thamm, P. Waltereit, K.H. Ploog, J. Appl. Surf. Sci. 175 (2001) 419.

[5] S.V. Novikov, C.T. Foxon, A.J. Kent, Phys. Status. Solidi C. 8 (2011) 1439.

[6] O. Ambacher, J. Majewski, C. Miskys, A. Link, M. Hermann, M. Eickhoff, M. Stutzmann, F. Bernardini, V. Fiorentini, V. Tilak, B. Schaff, L.F. Eastman, J. Phys. Condens. Matter 14 (2002) 3399.

[7] P. Saengkaew, S. Sanorpim, V. Yordsri, C. Thanachayanont, K. Onabe, J. Cryst. Growth 411 (2015) 76

[8] I.H. Oguzman, E. Bellotti, K.F. Brennan, J. Appl. Phys. 81 (1997) 7827.

[9] H. Vilchis, V.M. Sanchez-R, Mater. Sci. Semicond. Process. 37 (2015) 68.

[10] D. Xu, H. Yang, J.B. Li, S.F. Li, Y.T. Wang, D.G. Zhao, R.H. Wu, J. Cryst. Growth 206 (1999) 150.

[11] H. Chen, Z.Q. Li, H.F. Liu, L. Wan, M.H. Zhang, Q. Huang, J.M. Zhou, Y. Luo, Y.J. Han, K. Tao, N. Yang, J. Cryst. Growth 210 (2000) 811. 
[12] B. Yang, O. Brandt, A. Trampert, B. Jenichen, K.H. Ploog, J. Appl. Surf. Sci. 123 (1998) 1

[13] T. Onuma, T. Nozaka, H. Yamaguchi, T. Suzuki, S.F. Chichibu, J. Cryst. Growth 298 (2007) 193.

[14] S.F. Chichibu, M. Sugiyama, T. Nozaka, T. Suzuki, T. Onuma, K. Nakajima, T. Aoyama, M. Sumiya, T. Chikyow, A. Uedono, J. Cryst. Growth 272 (2004) 481.

[15] N. Zainal, S.V. Novikov, A.V. Akimov, C.R. Staddon, C.T. Foxon, A.J. Kent, Phys. B 407 (2012) 2964

[16] H. Tachibana, T. Ishido, M. Ogawa, M. Funato, S. Fujita, S. Fujita, J. Cryst. Growth 196 (1999) 41

[17] Z.X. Qin, H. Nagano, Y. Sugure, A.W. Jia, M. Kobayashi, Y. Kato, A. Yoshikawa, K. Takahashi, J. Cryst. Growth 189 (1998) 425.

[18] J. Laifi, N. Chaaben, H. Bouazizi, N. Fourati, C. Zerrouki, Y. El Gmili, A. Bchetnia, J.P. Salvestrini, B. El Jani, Superlattices Microstruct. 86 (2015) 472.

[19] A. Nakadaira, H. Tanaka, J. Electron. Mater. 26 (1997) 320.

[20] S. Suandon, S. Sanoprim, K. Yoodee, K. Onabe, Thin Solid Films 515 (2007) 4393.

[21] J. Laifi, N. Chaaben, H. Bouazizi, N. Fourati, C. Zerrouki, Y. El Gmili, A. Bchetnia, J.P. Salvestrini, B. El Jani, Superlattices Microstruct 94 (2016) 30.

[22] S. Strite, M.E. Lin, H. Morkoc,, Thin Solid Films 231 (1993) 197.

[23] R. Katayama, K. Onabe, Y. Shiraki, Phys. Status. Solidi (b) 241 (2004) 2739.

[24] J. Neugebauer, C. G. V. d Walle, Appl. Phys. Lett. 69 (1996) 3.

[25] J.L. Lyons, A. Janotti, C.G. Van de Walle, Appl. Phys. Lett. 97 (2010) 152108.

[26] D.O. Demchenko, I.C. Diallo, M.A. Reshchikov, Phys. Rev. Lett. 110 (2013) 087404.
[27] M. Iwinska, T. Sochacki, M. Amilusik, P. Kempisty, B. Lucznik, M. Fijalkowski, E. Litwin-Stazewska, J. Smalc-Koziorowska, A. Khapuridze, G. Staszczak, I. Grzegory, J. Cryst. Growth 456 (2016) 91.

[28] D.D. Koleske, A.E. Wichkenden, R.L. Henry, M.E. Twigg, J. Cryst. Growth 242 (2002) 55.

[29] D.J. As, U. Kohler, J. Phys. Condens. Matter 13 (2001) 8923.

30] A.F. Wright, J. Appl. Phys. 92 (2002) 2575.

31] O. Maksimova, Y. Gongb, H. Duc, P. Fisherc, M. Skowronskic, I.L. Kuskovskyb, V.D. Heydemanna, Vacuum 80 (2006) 1042

[32] K.W. Mah, J.-P. Mosnier, E. McGlynn, M.O. Henry, D. O'Mahony, J.G. Lunney, Appl. Phys. Lett. 80 (2002) 3310

[33] M. Godlewski, E.M. Goldys, M.R. Phillips, K. Pakuła, J.M. Baranowski, J. Appl. Surf. Sci. 177 (2001) 22

[34] O. Martinez, M. Mazzoni, F. Rossi, N. Armani, G. Salviati, P.P. Lottici, D. Bersani, Phys. Status. Solidi (a) 195 (2004) 26.

[35] K. Knobloch, P. Perlin, J. Krueger, E.R. Weber, C. Kisielowski, MRS Internet J. Nitride Semicond. Res. 3 (1998) 4.

[36] L. Liu, J.H. Edgar, Mater. Sci. Eng. 37 (2002) 61.

[37] F. Ben Nasr, A. Matoussi, R. Salh, S. Guermazi, H.J. Fitting, Z. Fakhfakh, Phys. E 41 (2009) 454.

[38] E. Deleporte, C. Guénaud, M. Voos, B. Beaumont, P. Gibart, Phys. Status. Solidi (b) 216 (1999) 713.

[39] G. Salviati, O. Martinez, M. Mazzoni, F. Rossi, N. Armani, P. Gucciardi, A. Vinattieri, D. Alderighi, M. Colocci, M.A. Gonzalez, J. Phys. Condens. Matter 14 (2002) 133. 